COMUNICAÇÃOCIENTÍFICA

\title{
TOLERÂNCIA À DESSECAÇÃO E INFLUÊNCIA DO TEGUMENTO NA GERMINAÇÃO DE SEMENTES DE CITRUMELO 'SWINGLE' (Citrus paradisi MACF X Poncirus trifoliata (L) RAF.) ${ }^{1}$

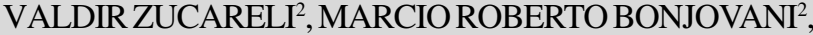 \\ CLÁUDIO CAVARIANI ${ }^{3}$, JOÃO NAKAGAWA ${ }^{3}$
}

RESUMO - O trabalho teve como objetivo estudar a tolerância à dessecação e a influência do tegumento na germinação de sementes de citrumelo 'Swingle'. As sementes foram extraídas manualmente e, em seguida, foi determinado o grau de umidade das sementes. Foi retirada uma amostra referente ao tratamento com o maior grau de umidade (48\%) a ser estudado, e as demais foram submetidas à secagem em estufa com circulação forçada de ar $\left(32 \pm 2^{\circ} \mathrm{C}\right)$, visando à obtenção dos outros tratamentos com diferentes graus de umidade. $\mathrm{O}$ teste de germinação foi instalado em delineamento experimental inteiramente casualizado, num fatorial 6x2 (grau de umidade x presença ou ausência de tegumento), com quatro repetições de 25 sementes por parcela. Após a obtenção de cada tratamento as sementes foram tratadas com o fungicida Thiabendazole $\left(0,4 \mathrm{~g} \cdot \mathrm{kg}^{-1}\right)$, semeadas em folhas de papel toalha umedecidas e confeccionados rolos que foram mantidos em câmara de germinação a $25^{\circ} \mathrm{C}$ sob luz constante. As avaliações foram realizadas a cada sete dias até o $35^{\circ}$ dia, sendo determinadas as porcentagens de germinação na primeira contagem, plântulas anormais, sementes mortas, sementes dormentes e germinação total. Também foram calculados o tempo médio e o índice de velocidade de germinação (IVG). Os dados foram submetidos à análise de variância, e as médias, comparadas pelo teste Tukey, a 5\% de probabilidade. As sementes toleraram a dessecação até baixos níveis de umidade (16\%), e a retirada do tegumento favoreceu o processo germinativo em sementes de citrumelo 'Swingle'.

Termos para indexação: Sementes recalcitrantes, porta-enxerto, citricultura.

\section{TOLERANCE TO DESICCATION AND INTEGUMENT INFLUENCE ON THE GERMINATION OF SWINGLE CITRUMELO (Citrus paradisi MACF X Poncirus trifoliata (L) RAF.) SEEDS}

\begin{abstract}
The current work aimed to study the tolerance to desiccation and the integument influence on the germination of seeds of Swingle citrumelo. Seeds were manually extracted and their humidity degree was determined. A sample of the group showing the highest humidity degree $(48 \%)$ was separated to be studied and the remaining samples were subjected to desiccation in an oven with forced air circulation $\left(32 \pm 2^{\circ} \mathrm{C}\right)$ in order to obtain other groups with different humidity degrees. Germination test was established in completely randomized experimental design, in a $6 \times 2$ factorial scheme (humidity degree $\mathrm{x}$ integument absence) with four replicates of 25 seeds per plot. After obtaining each group, seeds were treated with fungicide Thiabendazole $\left(0.4 \mathrm{~kg} \cdot \mathrm{g}^{-1}\right)$, sown in moist paper towels forming rolls, which were kept in a germination chamber at $25^{\circ} \mathrm{C}$ under constant light. The percentages of the first count germination, normal seedlings, dead seeds, dormant seeds and total germination were determined every seven days until the $35^{\text {th }}$ day. Germination mean time and speed germination index (IVG) were also calculated. Data were subjected to variance analysis and the means were compared by the Tukey test at $5 \%$ probability. The seeds tolerated the desiccation in low humidity levels and the integument removal favored the germination process in Swingle citrumelo seeds.
\end{abstract}

Index terms: Recalcitrant seeds, grafting stock, citriculture.

${ }^{1}$ (Trabalho 059-08). Recebido em:14-03-2008. Aceito para publicação em: 24-10-2008.

${ }^{2}$ Departamento de Botânica, Instituto de Biociências - Unesp - Botucatu-SP. CP. 510, CEP 18618-000, fone: (14) 38116265. E-mail: valdirzucareli@yahoo.com.br

${ }^{3}$ Departamento de Agricultura e Melhoramento Vegetal, FCA - Unesp - Botucatu-SP. CP 237, CEP 18903-970, fone :(14) 3811-7161. 
O citrumelo 'Swingle', um híbrido obtido do cruzamento de Citrus paradisi Macf. e Pancirus trifoliata (L) Raf., é considerado resistente à morte súbita dos citros (MSC), tolerante à gomose (Phytophthora sp.), ao nematóide dos citros (Tylenchulus semipenetrans), à tristeza dos citros, ao declínio, à exocorte, à xiloporose e a baixas temperaturas (Pompeu Júnior, 1991) e, quando utilizado como porta-enxerto, favorece a produção de frutos de alta qualidade e induz maturação dos frutos mais tardia que a apresentada pelo limão Cravo (Mattos Junior et al., 2005).

Nos últimos anos, tem ocorrido um grande interesse no citrumelo 'Swingle' para produção de mudas cítricas no Estado de São Paulo, com aumento de $20 \%$ no número de mudas produzidas sobre este porta-enxerto no período de 1999 a 2003 (Pompeu Júnior et al., 2004).

A obtenção do porta-enxerto é normalmente feita a partir de sementes, porém Ono et al. (1995) relatam que as sementes do citrumelo 'Swingle' apresentam baixa germinação. Este fato pode estar ligado à recalcitrância das sementes, pois King \& Roberts (1979) classificaram as sementes de citros como recalcitrantes e intermediárias.

Sementes que não toleram dessecação, a graus de umidade entre $15 \%$ e $20 \%$, são classificadas como recalcitrantes (Roberts, 1973). Embora, por definição, a secagem de sementes recalcitrantes resulte no declínio da viabilidade, na literatura tem sido reportada variação considerável na sensibilidade à dessecação (Fonseca \& Freire, 2003). Considerando essa variação, entre outras características, Farrant et al. (1988) propuseram a separação das sementes recalcitrantes em "altamente","moderadamente" e "minimamente" recalcitrantes.

Fortes (2002) e Oliveira \& Scivittaro (2007) obtiveram maior germinação em sementes de Poncirus trifoliata (L.) Raf. com a retirada do tegumento. Segundo Bewley \& Black (1994), o tegumento da semente pode conter várias substâncias capazes de interferir no processo de germinação, tais como fenóis, ceras e substâncias inibidoras da germinação. Também pode constituirse em obstáculo para o crescimento embrionário, regulando a disponibilidade de água para a embebição, interferindo nas trocas gasosas e na saída de substâncias inibidoras do embrião, formando uma barreira entre o embrião e o ambiente.

De acordo com o exposto, este trabalho objetivou estudar a tolerância à dessecação e influência do tegumento na germinação de sementes de citrumelo 'Swingle' (Citrus paradisi Macf X

\section{Poncirus trifoliata (L) Raf.).}

O experimento foi realizado no Laboratório de Análise de Sementes do Departamento de Produção Vegetal da Faculdade de Ciências Agronômicas (FCA) - Unesp - Câmpus de Botucatu.

Frutos maduros de citrumelo - 'Swingle' foram coletados no final do mês de abril de 2005, na Fazenda Morrinhos, propriedade da Botucatu-Cítrus, localizada no município de Botucatu-SP.

As sementes foram extraídas dos frutos manualmente, lavadas em água corrente e friccionadas contra uma peneira de malha de aço para a eliminação da mucilagem e de sementes malformadas ou abaixo do tamanho médio. A seguir, foi determinado o grau de umidade (ISTA, 2004) das sementes e retirada amostra para constituir o tratamento com o maior grau de umidade (48\%) a ser estudado. As sementes remanescentes foram acondicionadas em peneiras e submetidas à secagem, em estufa com circulação forçada de ar $\left(32 \pm 2^{\circ} \mathrm{C}\right)$, visando à obtenção dos demais tratamentos.

Os tratamentos de grau de umidade foram obtidos através do acompanhamento da perda de água das sementes durante a desidratação na estufa pela avaliação da massa a intervalos regulares. Conhecida a massa inicial úmida, as massas finais das amostras, correspondentes a cada um dos tratamentos desejados, foram calculadas utilizando a equação descrita por Hong \& Ellis (1996).

À medida que foram atingidas as massas correspondentes aos graus de umidade desejados, amostras foram retiradas, homogeneizadas e divididas em frações para a determinação do grau de umidade. A determinação foi realizada a $103 \pm 2^{\circ} \mathrm{C}$, durante 17 horas, pelo método da estufa (ISTA, 2004), utilizando-se de duas repetições de 25 sementes. Os resultados obtidos, com base na massa úmida, foram expressos em porcentagem.

As amostras correspondentes aos seis tratamentos, com diferentes graus de umidade, foram divididas em duas subamostras de sementes, uma mantida com tegumento e a outra sem, por remoção manual.

O teste de germinação foi instalado em delineamento experimental inteiramente casualizado, num fatorial 6x2 (grau de umidade x presença ou ausência de tegumento), com quatro repetições de 25 sementes por parcela. Foram testados seis graus de umidade das sementes $(48 \%, 43 \%, 35 \%, 28 \%$, $16 \%$ e $13 \%$ ) e duas condições de tegumento (com tegumento e sem tegumento).

As sementes foram tratadas com fungicida Thiabendazole $\left(0,4 \mathrm{~g} \cdot \mathrm{kg}^{-1}\right)$ e semeadas em folhas de papel toalha (rolo) umedecidas em volume de água 
equivalente a 2,5 vezes a sua massa sem hidratação. Os rolos foram mantidos em câmara de germinação a $25^{\circ} \mathrm{C}$ sob luz constante. As avaliações foram realizadas a cada sete dias até o $35^{\circ}$ dia, sendo determinadas as porcentagens de germinação na primeira contagem, plântulas anormais, sementes mortas e dormentes, e germinação total (Brasil, 1992). Também foram calculados o tempo médio de germinação (Labouriau, 1983) e o índice de velocidade de germinação (Maguire, 1962).

Os dados foram submetidos à análise de variância, e as médias, comparadas pelo teste Tukey, a $5 \%$ de probabilidade. Para os dados em porcentagem, foi utilizada a transformação arcoseno da raiz quadrada de $\mathrm{x} / 100$, e para os dados numéricos, a transformação raiz quadrada de $\mathrm{x}+0,5$.

Todas as variáveis analisadas, exceto o tempo médio de germinação, foram afetadas pela interação dos fatores avaliados, cujos desdobramentos são apresentados na Tabela 1.

As maiores médias para porcentagem de germinação foram obtidas a partir de sementes sem tegumento, exceto nos tratamentos com $35 \%$ e $13 \%$ de umidade. Resultados semelhantes foram observados por Fortes (2002) em sementes de Poncirus trifoliata (L.) Raf., que verificou maior porcentagem de germinação quando com a retirada do tegumento. Oliveira \& Scivittaro (2007) também observaram que a remoção do tegumento favoreceu a germinação de sementes de Poncirus trifoliata (L.) Raf. em relação ao uso de semente com tegumento íntegro.

De acordo com Labouriau (1983), a ruptura ou retirada do tegumento pode induzir a mudanças, como aumento da permeabilidade aos gases e água, a sensibilidade à luz ou temperatura, bem como a remoção de substâncias inibidoras, o que justificaria o melhor desempenho das sementes sem tegumento.

Embora não significativo estatisticamente, observa-se que as sementes com tegumento tiveram a porcentagem de germinação favorecida em nove pontos percentuais quando secadas ao nível de $35 \%$ de umidade (Tabela 01). O beneficio sobre a germinação após redução moderada do grau de umidade também foi relatado por Martins \& Silva (2006) em sementes de tangerina (Citrus reticulata Blanco), que apresentaram aumento de $7 \%$ quando o nível de umidade foi reduzido de $49 \%$ para $39 \%$ (72,5 e 85,5\% de germinação, respectivamente).

Maiores médias para porcentagem de sementes mortas e dormentes foram observadas à medida que se diminuiu o grau de umidade das sementes. Neste aspecto, Nautiyal \& Purohit (1985) explicaram que a perda de água em sementes recalcitrantes desencadeia alguns processos deterioráveis, como a desnaturação de proteínas, alterações na atividade das enzimas peroxidases $\mathrm{e}$ danos no sistema de membranas, podendo resultar na completa perda de sua viabilidade. $\mathrm{O}$ aumento de sementes dormentes pode estar relacionado com mecanismos de dormência secundária, que se instala em sementes quiescentes, após a dispersão, quando estas encontram ambiente desfavorável ou estressantes, neste caso há dessecação (Cardoso, 2004).

Não houve interferência do grau de umidade no tempo médio de germinação, no entanto sementes sem tegumento apresentaram menor tempo de germinação quando comparadas às sementes com tegumento.

Os maiores índices de velocidade de germinação (IVG) foram obtidos nas sementes sem tegumento, exceto para o tratamento com o menor grau de umidade avaliado (13\%). A maior velocidade de germinação em sementes sem tegumento foi confirmada pela maior porcentagem de plântulas normais obtidas na ocasião da primeira contagem (Tabela 01).

Os maiores índices de velocidade de germinação obtidos em sementes sem tegumento podem estar relacionados com a resistência oferecida pelo tegumento à expansão do embrião, citada por Toledo e Marcos Filho (1977), e, também, pela permeabilidade do tegumento, que pode influenciar na velocidade de embebição das sementes como mencionado por Toledo \& Marcos Filho (1977) e Carvalho \& Nakagawa (2000).

$\mathrm{O}$ fato de as sementes com tegumento e menor grau de umidade $(13 \%)$ serem favorecidas na germinação e no IVG demonstra a importância do tegumento na troca de água com o ambiente. Tais resultados podem estar relacionados com a velocidade de embebição, pois, segundo Marcos Filho (2005), quando as sementes apresentam baixo teor de água, a rápida embebição pode ocasionar em rompimento das membranas celulares; nesse caso o tegumento atuaria de forma a diminuir a velocidade de embebição e evitar tais danos. McDonald et al. (1988) também mencionaram que o tegumento é um fator regulador da embebição da semente.

Conclui-se que as sementes toleram a dessecação até baixos níveis de umidade e que a retirada do tegumento favoreceu o processo germinativo em sementes de citrumelo 'Swingle'. 
TABELA 1 - Porcentagem de germinação na primeira contagem (PC), germinação total (GT), plântulas anormais (AN), sementes mortas (SM) e dormentes (SD), tempo médio de germinação (TMG) e índice de velocidade de germinação (IVG) em sementes de citrumelo 'Swingle' em função do grau de umidade e presença de tegumento. Botucatu-SP, 2005.

\begin{tabular}{|c|c|c|c|c|c|c|c|c|c|c|c|c|c|}
\hline & \multicolumn{2}{|c|}{$\begin{array}{l}\text { PC } \\
(\%)\end{array}$} & \multicolumn{2}{|c|}{$\begin{array}{l}\text { GT } \\
(\%)\end{array}$} & \multicolumn{2}{|c|}{$\begin{array}{l}\text { AN } \\
(\%)\end{array}$} & \multicolumn{2}{|c|}{$\begin{array}{l}\text { SM } \\
(\%)\end{array}$} & \multicolumn{2}{|c|}{$\begin{array}{l}\text { SD } \\
(\%)\end{array}$} & \multicolumn{2}{|c|}{$\begin{array}{l}\text { TMG } \\
\text { (dias) }\end{array}$} & IVG \\
\hline Umidade (\%) & $\mathrm{S} / \mathrm{T}$ & $\mathrm{C} / \mathrm{T}$ & $\mathbf{S} / \mathbf{T}$ & $\mathrm{C} / \mathrm{T}$ & $\mathbf{S} / \mathbf{T}$ & $\mathrm{C} / \mathrm{T}$ & $\mathbf{S} / \mathbf{T}$ & $\mathrm{C} / \mathrm{T}$ & $\mathbf{S} / \mathbf{T}$ & $\mathrm{C} / \mathrm{T}$ & $\mathbf{S} / \mathbf{T}$ & $\mathbf{C} / \mathbf{T}$ & $\mathrm{C} / \mathrm{T}$ \\
\hline 48 & $61 \mathrm{Aa}$ & $5 \mathrm{~B} \mathrm{ab}$ & $94 \mathrm{~A} \mathrm{a}$ & $63 \mathrm{~B} \mathrm{ab}$ & $4,0 \mathrm{~A} a b$ & $3,0 \mathrm{~A} \mathrm{a}$ & $1 \mathrm{~B} \mathrm{~cd}$ & $6 \mathrm{Ab}$ & $1 \mathrm{~B} \mathrm{ab}$ & $29 \mathrm{~A} \mathrm{a}$ & 10,50 & 23,75 & $2,73 \mathrm{~A}$ a $0,85 \mathrm{~B}$ abc \\
\hline 43 & $56 \mathrm{~A} \mathrm{a}$ & $13 \mathrm{~B}$ & $93 \mathrm{~A} \mathrm{a}$ & $61 \mathrm{~B} \mathrm{a} \mathrm{b}$ & $6,0 \mathrm{~A} \mathrm{ab}$ & $4,0 \mathrm{~A} \mathrm{a}$ & $0 \mathrm{Ad}$ & $3 \mathrm{Ab}$ & $1 \mathrm{~B} \mathrm{ab}$ & $30 \mathrm{~A} \mathrm{a}$ & 11,50 & 18,75 & $2,75 \mathrm{~A}$ a $1,07 \mathrm{~B}$ a \\
\hline 35 & $49 \mathrm{~A} \mathrm{a}$ & $2 \mathrm{~B} \mathrm{~b}$ & $81 \mathrm{Aa}$ & $72 \mathrm{~A} \mathrm{a}$ & $8,0 \mathrm{Aa}$ & $2,0 \mathrm{Ba}$ & $5 \mathrm{~A} \mathrm{bcd}$ & $6 \mathrm{Ab}$ & $6 \mathrm{~B} \mathrm{ab}$ & $20 \mathrm{~A} \mathrm{ab}$ & 11,25 & 24,50 & $2,34 \mathrm{~A}$ a $0,97 \mathrm{~B}$ ab \\
\hline 28 & $17 \mathrm{Ab}$ & $0 \mathrm{~B} \mathrm{~b}$ & $80 \mathrm{~A} \mathrm{a}$ & $61 \mathrm{~B} \mathrm{ab}$ & $4,0 \mathrm{Aab}$ & $4,0 \mathrm{Aa}$ & $7 \mathrm{~A} \mathrm{bc}$ & $4 \mathrm{Ab}$ & $9 \mathrm{~B} \mathrm{a}$ & $31 \mathrm{~A} \mathrm{a}$ & 14,75 & 21,75 & 1,63 A b 0,83 B abc \\
\hline 16 & $8 \mathrm{~A} \mathrm{bc}$ & $0 \mathrm{~B} \mathrm{~b}$ & $79 \mathrm{~A} \mathrm{a}$ & $50 \mathrm{~B} \mathrm{a} \mathrm{b}$ & $6,0 \mathrm{Aa}$ & $4,0 \mathrm{Aa}$ & $9 \mathrm{~B} \mathrm{~b}$ & $31 \mathrm{~A} \mathrm{a}$ & $6 \mathrm{~B} \mathrm{ab}$ & $15 \mathrm{~A} \mathrm{ab}$ & 14,50 & 25,00 & $1,58 \mathrm{~A} \mathrm{~b} 0,63 \mathrm{~B} \mathrm{bc}$ \\
\hline 13 & $4 \mathrm{Ac}$ & $0 \mathrm{Ab}$ & $8 \mathrm{~B} \mathrm{~b}$ & $40 \mathrm{~A} \mathrm{~b}$ & $0,0 \mathrm{Bb}$ & $6,0 \mathrm{Aa}$ & $92 \mathrm{~A} \mathrm{a}$ & $44 \mathrm{~B} \mathrm{a}$ & $0 \mathrm{~B} \mathrm{~b}$ & $10 \mathrm{Ab}$ & 10,50 & 27,00 & $0,20 \mathrm{~B} \mathrm{c} 0,47 \mathrm{Ac}$ \\
\hline Média & 32,5 & 3,33 & 72,5 & 57,8 & 4,6 & 3,8 & 19 & 15,6 & 3,8 & 22,5 & $12,17 \mathrm{~B}$ & $23,46 \mathrm{~A}$ & 0,8 \\
\hline \multicolumn{14}{|l|}{ Valor F } \\
\hline Teg & \multicolumn{2}{|c|}{$184,06 * *$} & \multicolumn{2}{|c|}{$25,87 * *$} & \multicolumn{2}{|c|}{$1,00 \mathrm{~ns}$} & \multirow{2}{*}{\multicolumn{2}{|c|}{$0,81 \mathrm{~ns}$}} & \multicolumn{2}{|c|}{$94,00^{* *}$} & \multicolumn{2}{|c|}{$68,90 * *$} & $217,92 * *$ \\
\hline Grau Umidade & \multicolumn{2}{|c|}{$33,22 * *$} & \multicolumn{2}{|c|}{$36,17 * *$} & \multicolumn{2}{|c|}{$1,10 \mathrm{~ns}$} & & & \multicolumn{2}{|c|}{$4,38 * *$} & \multicolumn{2}{|c|}{$0,91 \mathrm{~ns}$} & $56,77 * *$ \\
\hline Teg XGrau & \multicolumn{2}{|c|}{$7,67 * *$} & \multicolumn{2}{|c|}{$15,20 * *$} & \multicolumn{2}{|c|}{$3,44^{*}$} & \multicolumn{2}{|c|}{$9,72 * *$} & \multirow{2}{*}{\multicolumn{2}{|c|}{$\frac{3,23 *}{31,49}$}} & & & $21,30^{* *}$ \\
\hline C.V.(\%) & \multicolumn{2}{|c|}{33,85} & \multicolumn{2}{|c|}{12,42} & \multicolumn{2}{|c|}{59,74} & \multicolumn{2}{|c|}{26,51} & & & \multicolumn{2}{|c|}{13,8} & 6,49 \\
\hline
\end{tabular}

Médias seguidas de letras iguais, maiúsculas na linha e minúsculas na coluna, não diferem entre si, pelo teste Tukey.

* - significativo a $5 \%$ de probabilidade.

** - significativo a $1 \%$ de probabilidade.

$\mathrm{S} / \mathrm{T}$ e $\mathrm{C} / \mathrm{T}$ - sem tegumento e com tegumento, respectivamente.

\section{REFERÊNCIAS}

BEWLEY, J.D; BLACK. M. Seeds: physiology of development and germination. New York: Plenum Press, 1994. 445p.

BRASIL. Ministério da Agricultura. Departamento Nacional de Produção Vegetal. Divisão de Sementes e Mudas. Regras para análise de sementes. Brasília, 1992. 188p.

CARDOSO, V.J.M. Dormência: estabelecimento do processo. In: FERREIRA, A.G; BORGHETTI, F. (Org.). Germinação: do básico ao aplicado. Porto Alegre: Artimed, 2004. p.95-108.

CARVALHO, N.M.; NAKAGAWA, J. Sementes: ciência, tecnologia e produção. 4.ed. Jaboticabal: Funep, 2000. 588p.

FARRANT, J.M.; PAMMENTER, N.W.; BERJAK, P. Recalcitrance: a current assessment. Seed Science and Technology, Zurich, v.16, p.155-166, 1988.

FONSECA, S.C.L.; FREIRE, H.B. Sementes recalcitrantes: problemas na pós-colheita. Bragantia, Campinas, v.62, n.2, p.297-303, 2003.

FORTES, A.M.T. Efeito de reguladores vegetais na propagação de Poncirus Trifoliata (L.) Raf. var. monstrosa, Flying Dragon. 98f. 2002. Tese (Doutorado em Ciências Biológicas - Fisiologia
Vegetal) - Instituto de Biociências, Universidade Estadual Paulista, Botucatu, 2002.

HONG, T.D.; ELLIS, R.H. A protocol to determine seed storage behavior. Rome: International Plant Genetic Resources Institute, 1996. 55p. (Technical Bulletin,1).

ISTA - INTERNATIONAL SEED TESTING ASSOCIATION. Determination of moisture content. Bassersdorf, 2004. p.9.1-9.8.

KING, M.W.; ROBERTS, E.H. The storage of recalcitrant seeds: achievements and possible approaches. Rome: IBPGR, 1979. 22p.

LABOURIAU, L.G. A germinação de sementes. Washington: Organização dos Estados Americanos, 1983. 174p.

MAGUIRE, J.D. Speed of germination-aid in relation evaluation for seedling emergence vigor. Crop Science, Madison, v.2, n.2, p.176-177, 1962.

MARCOS-FILHO, J. Fisiologia de sementes de plantas cultivadas. Piracicaba: FEALQ, 2005. 495p.

MARTINS, L.; SILVA, W.R. Comportamento fisiológico de sementes de tangerina (Citrus reticulata Blanco) submetidas à desidratação. Revista Brasileira de Fruticultura, Jaboticabal, v.28, n.1, p.8-10, 2006. 
MATTOS JUNIOR, D.; NEGRI, J.D.; PIO, R.M.; POMPEU JUNIOR, J. (Org.). Citros. CordeiropolisSP: Centro APTA Citros Sylvio Moreira, 2005. v.1, $929 \mathrm{p}$.

McDONALD, M.B.; VERTUCCI, C.W.; ROOS, E.E. Seed coat regulation of soybean seed imbibition. Crop Science, Madison, v.28, n.6, p.987-992, 1988.

NAUTIYAL, A.R.; PUROHIT, A.N. Seed viability in sal. II. Physiological and biochemical aspects of ageing in seeds of Shorea robusta. Seed Science and Technology, Zurich, v.13, p.69-76, 1985.

OLIVEIRA, R.P.; SCIVITTARO, W.B. Formação do porta-enxerto Trifoliata: época de semeadura e tegumento na emergência de plântulas. Ciência Rural, Santa Maria, v.37, n.1, p.281-283, 2007.

ONO, E.O.; LEONEL, S.; RODRIGUES, J.D. Efeito de fitorreguladores na germinação de sementes de citrumelo 'Swingle'. Semina, Londrina, v.16, n.1, p.4750,1995
POMPEU JÚNIOR, J. Porta-enxertos. In: In: RODRIGUEZ, O.; VIEGAS, F.; POMPEU JÚNIOR, J.; AMARO, A.A. (Ed.). Citricultura brasileira. Campinas: Fundação Cargill, 1991. p.265-280.

POMPEU JÚNIOR, J.; SALVA, R.; BLUMER, S. Copas e porta-enxertos nos viveiros de mudas cítricas do Estado de São Paulo. Laranja, Cordeirópolis, v.25, n.2, p.413-422, 2004.

ROBERTS, E.H. Predicting the storage life of seeds. Seed Science and Tecnology, Zurich, v.1, p.499-514, 1973.

TOLEDO, F. F.; MARCOS FILHO, J. Manual das sementes: tecnologia da produção. São Paulo: Agronômica Ceres, 1977. 224p. 